\title{
RESULTADOS DE LA CATALOGACIÓN DE LOS SCARABAEOIDEA LAPAROSTICTI (INSECTA, COLEOPTERA) COPRÓFAGOS DE MARRUECOS Y EL SÁHARA OCCIDENTAL CONSERVADOS EN LAS COLECCIONES DEL MUSEO NACIONAL DE CIENCIAS NATURALES
}

\author{
J. Romero Samper ${ }^{1}$, F. J. Cabrero-Sañudo², J. M. Lobo³ , C. Martín \\ Albaladejo $^{3} \&$ I. Izquierdo Moya ${ }^{3}$
}

\section{RESUMEN}

J. Romero Samper, F. J. Cabrero-Sañudo, J. M. Lobo, C. Martín Albaladejo \& I. Izquierdo Moya. 2011. Resultados de la catalogación de los Scarabaeoidea Laparosticti (Insecta, Coleoptera) coprófagos de Marruecos y el Sáhara Occidental conservados en las colecciones del Museo Nacional de Ciencias Naturales. Grael/sia, 67(1): 47-56.

Se ofrece el listado pormenorizado de las 120 especies de Scarabaeoidea coprófagos de Marruecos y el Sáhara Occidental (familias Aphodiidae, Geotrupidae y Scarabaeidae), depositadas en la colección de Entomología del Museo Nacional de Ciencias Naturales de Madrid (Consejo Superior de Investigaciones Científicas), resultado de la catalogación del material presente en esta institución. Asimismo, se aportan mapas con la localización de dichos registros y se realiza un breve resumen histórico de los colectores y la procedencia de este material, examinando su evolución temporal y analizando la representatividad del mismo respecto al catálogo de especies conocidas de Marruecos para este grupo.

Palabras clave: Coleoptera; Scarabaeoidea; coprófagos; Marruecos; Sáhara Occidental; Museo Nacional de Ciencias Naturales; colecciones entomológicas.

\begin{abstract}
J. Romero Samper, F. J. Cabrero-Sañudo, J. M. Lobo, C. Martín Albaladejo \& I. Izquierdo Moya. 2011. Results of the cataloguing of the Scarabaeoidea Laparosticti dung beetles (Insecta, Coleoptera) from Morocco and Western Sahara preserved in the collections of the Museo Nacional de Ciencias Naturales. Grael/sia, 67(1): 47-56 (in Spanish).

We offer a species list containing the 120 dung beetle species from Morocco and Western Sahara (families Aphodiidae, Geotrupidae and Scarabaeidae), deposited in the Entomological collection of the Museo Nacional de Ciencias Naturales (Consejo Superior de Investigaciones Científicas).We also include several maps showing the collection sites and a brief historical summary about the collectors and the origin of the specimens, examining their temporal evolution and analyzing their representativeness compared with the catalogue of species belonging to this group known from Morocco.
\end{abstract}

Key words: Coleoptera; Scarabaeoidea; dung beetles; Morocco; Western Sahara; Museo Nacional de Ciencias Naturales; entomological collections.

Departamento de Psicología. Facultad de Medicina, Universidad CEU San Pablo. Campus de Montepríncipe, edificio C. 28668 Boadilla del Monte, España - jrsamper@ceu.es

2 Departamento de Zoología y Antropología Física, Facultad de Ciencias Biológicas, Universidad Complutense de Madrid. José Antonio Novais, 2, Ciudad Universitaria, 28040 Madrid, España - fjcabrero@bio.ucm.es

3 Departamento de Biodiversidad y Biología Evolutiva, Museo Nacional de Ciencias Naturales (CSIC), C/José Gutiérrez Abacal 2, 28006 Madrid, España - mcnj117@mncn.csic - carolina.martin@mncn.csic.es - izquierdo@mncn.csic.es 


\section{Introducción y antecedentes históricos}

La fauna coleopterológica del norte de África ha atraído, desde siempre, la atención de los entomólogos europeos. No extraña tal predilección por un área exótica, dada su proximidad al viejo continente. La distancia menor entre ambas costas es el estrecho de Gibraltar, con apenas 14,4 kilómetros. Otro motivo de interés hacia esta región lo constituye el hecho histórico de que esos territorios estuvieron, hasta el siglo pasado, bajo la dominación colonial de distintas naciones europeas (España, Francia, Gran Bretaña e Italia), situación que facilitó la ejecución de expediciones y muestreos por parte de científicos y naturalistas europeos de los siglos XIX y XX. Estas mismas expediciones constituyen hoy en día, por sí mismas, otra de las razones del marcado interés existente aún por la zona.

Por lo que atañe a Marruecos y al Sáhara Occidental, ya desde el siglo XIX son reseñables algunas expediciones españolas al África noroccidental (Hernández-Pacheco et al., 1949) que, aunque no tuvieron originalmente carácter científico, realizaron capturas entomológicas entre otros fondos de historia natural. Cabe mencionar entre ellas (Conde-Salazar Infiesta, 2009) la de Julio Cervera, Francisco Quiroga y Felipe Rizzo en 1866 (Quiroga, 1886) al Adrar (Península del Río de Oro: Uad Meghetha Merzug), o la de Norberto Font y Sagué en 1902 a la Península del Río de Oro, así como la de Oskar Lenz y Cristóbal Benítez en 1880 (Benítez, 1899 [reeditada en 2005]) atravesando Marruecos y el Sáhara Occidental hasta Senegal, y la de Juan Dantín Cerceda, Ángel Cabrera Latorre y Constancio Bernaldo de Quirós en 1913 al protectorado español en Marruecos (Real Sociedad Española de Historia Natural, 1914; Cabrera, 1924; González Bueno \& Gomis Blanco, 2007).

Varios de los autores antes mencionados recolectaron algunos Scarabaeoidea coprófagos; así por ejemplo, entre 1933 y 1934, Cristóbal Benítez recolectó especímenes de las tres familias aquí tratadas. Pero, sin duda, las expediciones más fructíferas fueron las llevadas a cabo por Manuel Martínez de la Escalera y su hijo Fernando, sin desdeñar las aportaciones de José Arias Encobet, comisionado durante varios años por el Museo Nacional de Ciencias Naturales (MNCN) para la formación de colecciones zoológicas de las costas de Marruecos; Cándido Bolívar, que muestreó junto con varios colegas, como Juan Gil Collado y Federico Bonet, la zona del Rif en 1930; Joaquín Mateu Sempere realizó numerosas expediciones en el Sáhara español en la década de los 40 del pasado siglo; también en estos mismos años, Eugenio Morales Agacino realizó campañas entomológicas en el Sáhara español y alguna en la zona del protectorado español en Marruecos; José Giner Marí, con su prospección al Sáhara y la zona oriental de Marruecos en 1945; o, Antonio Cobos, que realizó varias campañas en la Yebala, Gomara y Rif, dentro del protectorado español en Marruecos, durante la década de los 50 del siglo XX (Arias Encobet, 1913; Bolívar, 1930; Giner Marí, 1944; Mateu, 1950; Cobos, 1955, 1961; Morales Agacino, 2001). Otros españoles que también recolectaron en la zona fueron Anselmo Pardo Alcalde (López-Colón \& Baena, 2005), Francisco Codina Padilla y Rafael Candel Vila.

Manuel Martínez de la Escalera realizó cuatro expediciones entomológicas en Marruecos antes de asentar su domicilio en Mogador: 1905, 1906, 1907 y 1909. A partir de entonces y hasta 1948, sus prospecciones fueron muy numerosas. Desde 1909 le acompañó ocasionalmente su hijo Fernando, quien llevó a cabo su última campaña en el Sáhara Occidental en 1935, en una misión comandada por Francisco Hernández-Pacheco de la Cuesta (Hernández-Pacheco et al., 1949). Fernando realizó también campañas de manera autónoma, sin su padre Manuel (Martínez de la Escalera, 1913, 1914). En las recolecciones de material, también le ayudó a Manuel su hijo menor "Lolo" (Real Sociedad Española de Historia Natural [C.B. Gómez de Quirós], 1914; González Bueno \& Gomis Blanco, 2007). Martínez de la Escalera (1914) colaboró, además, con otros colegas europeos: Buchet, Favier, Simon, Vaucher y Walker. Con más de quinientas páginas, el catálogo "Los Coleópteros de Marruecos", de Manuel Martínez de la Escalera \& Pérez de Rozas (1914), constituye la obra más extensa y meticulosa de aquellos inicios en el estudio de la fauna coleopterológica del África Paleártica. La obra tiene su origen en diversas expediciones fomentadas por la Comisión Permanente para el Estudio del Noroeste de África. Manuel Martínez de la Escalera prospectó vastas áreas de Marruecos (Martínez de la Escalera, 1914; Casado, 1998; López-Colón \& Baena, 2005) y su hijo Fernando, además, zonas del Sáhara Occidental. Entre otras de sus 150 publicaciones (Martín Albaladejo, 2005) cabe destacar la descripción de nuevos lamelicornios marroquíes y un buen número de inventariados faunísticos (Martínez de 


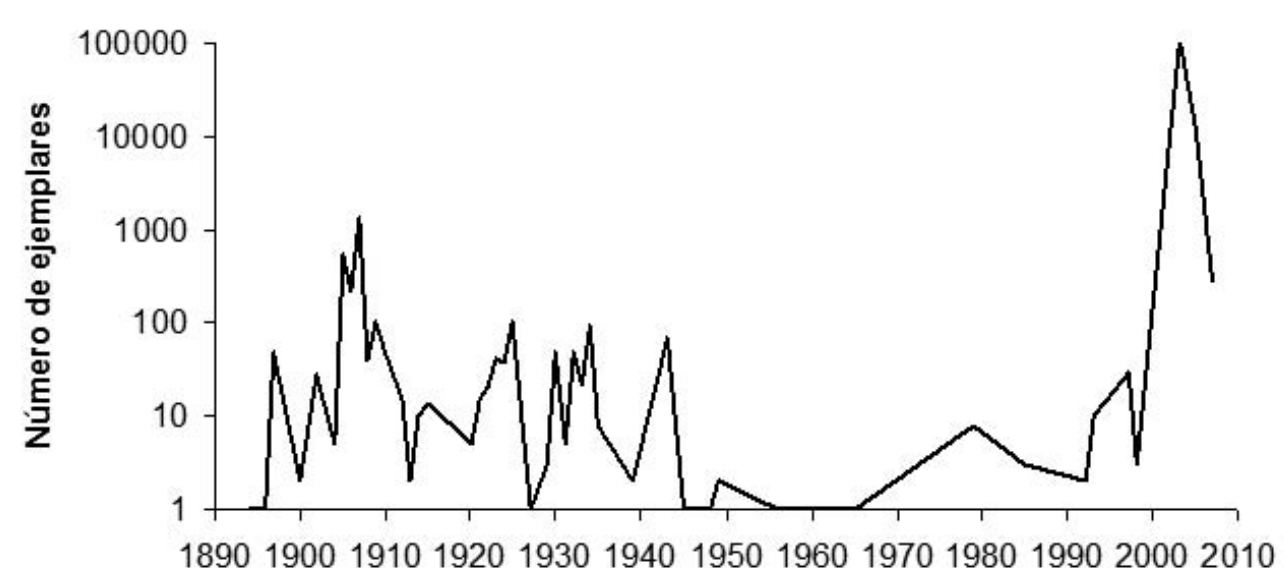

Año

Fig. 1.- Variación en el número total de ejemplares de Scarabaeoidea coprófagos (en escala logarítmica) conservados en la Colección de Entomología del Museo Nacional de Ciencias Naturales (MNCN) según el año de su colecta. Sólo se han consignado aquellos ejemplares con fecha de colecta conocida $(n=127.446)$.

Fig. 1.- Variation in the total number of specimens of Scarabaeoidea dung beetles (in logarithmic scale) preserved in the Collection of Entomology of the MNCN according to the year of his collection. Only those specimens with known date of collection have been recorded $(n=127.446)$.

la Escalera, 1906, 1913, 1914, 1925, 1926, 1931, 1933 y 1934).

Mención especial merecen las aportaciones de Anselmo Pardo Alcaide (López-Colón \& Baena, 2005), discípulo de Martínez de la Escalera, quien no sólo describió, en lo que se refiere a los Coleoptera Scarabaeoidea, tres especies de Glaresis Erichson, 1848 (Pardo Alcaide, 1958) y un afodino del Marruecos nororiental y sur de la Península Ibérica, Ahermodontus ambrosi Pardo Alcaide, 1936, sino que colectó buen número de ejemplares de Scarabaeoidea (Pardo Alcaide, 1950, 1955), no todos ellos etiquetados con su nombre como colector.

Tras un lapso cronológico (de 1945 a 2003, véase Fig. 1), durante el que apenas se incorpora material de estas especies a la colección, algunos de los autores de este trabajo participan en tres campañas $(2003,2005$ y 2007), que enriquecen notablemente las colecciones del MNCN y sirvieron para fundamentar la tesis de uno de los autores de este trabajo (Romero Samper, 2008). Estas tres campañas estuvieron restringidas únicamente al Medio Atlas Central y Occidental. Dicho material se con- serva mayoritariamente en alcohol y representa, aproximadamente, más del $94 \%$ del total de todos los ejemplares actualmente existentes (Tabla 1) y aproximadamente la mitad de las especies de Scarabaeoidea Laparosticti coprófagos marroquíes conservadas actualmente en las colecciones del MNCN. La abundancia del material colectado se debe, principalmente, a la reconocida eficacia de las trampas de caída cebadas para la colecta de estas especies (Lobo et al., 1988; Veiga et al., 1989).

El objetivo fundamental de este trabajo es, por tanto, presentar un listado de las especies de Scarabaeoidea coprófagas presentes en las colecciones del Museo Nacional de Ciencias Naturales, así como mostrar la distribución espacial y temporal de los registros del material conservado en dicha institución.

\section{Metodología y cobertura taxonómica y geográfica}

Mediante un proyecto financiado por el Instituto de Estudios Ceutíes (http://www.ieceuties.org/) se ha desarrollado una base de datos que recopila toda la 
Tabla 1.- Total de ejemplares recolectados por distintos autores, tabulados en orden alfabético e indicando los años de las campañas de colecta.

Table 1.- Total number of specimens gathered by different authors, tabulated in alphabetical order and indicating the years of the collection campaigns.

\begin{tabular}{|c|c|c|}
\hline Colector & Años de las colectas & Total ejemplares \\
\hline Ahrens, D. & 1992 & 2 \\
\hline Alvaneli y Théry, A. & 1935 & 6 \\
\hline Antoine, P. & 1920 & 6 \\
\hline Arias Encobet, J. & 1906-1909 & 94 \\
\hline Benítez, C. & 1933-1934 & 23 \\
\hline Bolívar, C. & 1930-1932 & 83 \\
\hline Boucher, S. & 1985 & 1 \\
\hline Cabrera Diaz, A. & 1921 & 8 \\
\hline Cabrero-Sañudo, F., Hortal, J., Jiménez Valverde, A., Lobo, J.M. y Romero Samper, J. & 2003-2005 & 124.222 \\
\hline Candel Vila, R. & 1927 & 17 \\
\hline Casares, A. & ¿? & 3 \\
\hline Cazurro Ruiz, M. & ¿? & 1 \\
\hline Ceballos, G. & ¿? & 2 \\
\hline Codina Padilla, F. & 1949-1956 & 33 \\
\hline Dorda, J. y Rodríquez, $\mathrm{R}$. & ¿? & 1 \\
\hline Fernández Rubio, F. & 1993 & 1 \\
\hline Gil, J. & 1923 & 20 \\
\hline Giner Mari, J. & 1943 & 9 \\
\hline Hortal, J., Jiménez Valverde, A. y Lobo, J.M. & 2007 & 285 \\
\hline Le Boul, M. & 1900-1909 & 5 \\
\hline Lozano, C. & ¿? & 34 \\
\hline Martín Albaladejo, C. & 1993 & 8 \\
\hline Martínez de la Escalera, F. & 1909-1934 & 1.183 \\
\hline Martínez de la Escalera, F. y Casares, A. & ¿? & 5 \\
\hline Martínez de la Escalera, F. & 1932-1935 & 65 \\
\hline Martínez de la Escalera, M. & $1905-1943$ & 5.223 \\
\hline Martín Piera. F. & 1997 & 24 \\
\hline Menor, C. & ¿? & 4 \\
\hline Nombela, A. & ¿? & 1 \\
\hline Pardo Alcaide, A. & $1915-1933$ & 4 \\
\hline Pau, C. & ¿? & 3 \\
\hline Pérez Sánchez, J. & ¿? & 1 \\
\hline Ruíz García, J.L. & 1997-1998 & 8 \\
\hline Théry, A. & ¿? & 1 \\
\hline Urquijo, $\mathrm{A}$. & 1948 & 1 \\
\hline
\end{tabular}

información taxonómica y biogeográfica disponible en la literatura y diversas colecciones entomológicas sobre los Scarabaeoidea Laparosticti de Marruecos. El conjunto de esta información está libremente disponible a través de http://www.biogeografia.org/ y para su compilación, entre otras fuentes consultadas, se revisó todo el material perteneciente a las familias Aphodiidae, Geotrupidae y Scarabaeidae depositado en las colecciones del MNCN, tanto en seco como el conservado en alcohol. Como bibliografía de consulta, se utilizaron las referencias de Baraud (1985, 1987) y Hollande \& Thérond (1998). Hay que tener en cuenta que una parte del material depositado en esta colección no conserva etiquetas en las que figuren sus recolectores, aunque cronológicamente o por la localidad de muestreo pueda inferirse la autoría del mismo. Es el caso de los ejemplares provenientes de las colectas realizadas por Martínez de la Escalera o Pardo Alcaide.

La colección de Coleoptera Scarabaeoidea coprófagos del MNCN (familias Scarabaeidae, Geotrupidae y Aphodiidae) está compuesta por 120 especies pertenecientes a 3 subfamilias, 10 tribus y 60 géneros (Tabla 2) y en ella se conservan (a enero de 2011) 131.907 ejemplares recolectados por 38 entomólogos (Tabla 1) en 196 localidades diferentes. Esta colección 
Tabla 2.- Relación de especies de Scarabaeoidea coprófagos conservados en la Colección de Entomología del Museo Nacional de Ciencias Naturales.

Table 2.- List of Scarabaeoidea dung beetles species preserved in the Collection of Entomology of the MNCN.

\begin{tabular}{|c|c|c|c|}
\hline Familia Subfamilia & Especie & Familia Subfamilia & Especie \\
\hline $\begin{array}{l}\text { Aphodiidae } \\
\qquad \text { Aphodiinae }\end{array}$ & 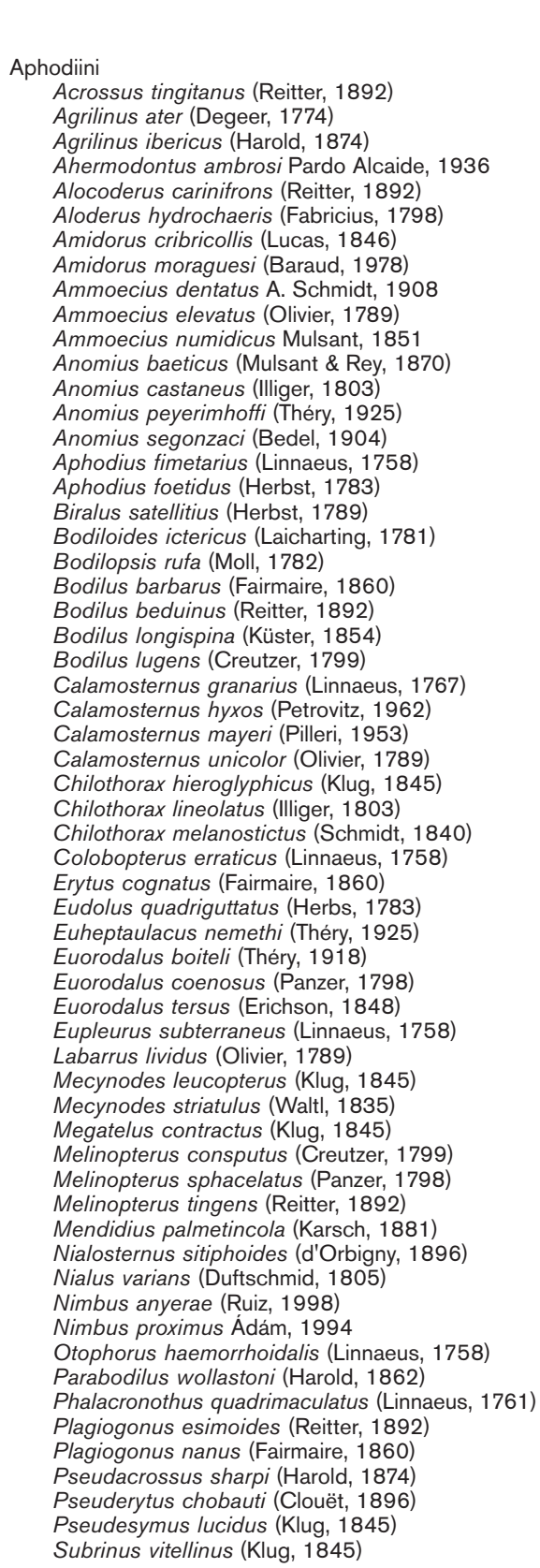 & $\begin{array}{l}\text { Scarabaeidae } \\
\text { Scarabaeinae }\end{array}$ & $\begin{array}{l}\text { e } \\
\text { Coprini } \\
\text { Copris hispanus (Linnaeus, 1764) } \\
\text { Oniticellini } \\
\text { Euoniticellus fulvus (Goeze, 1777) } \\
\text { Euoniticellus pallens (Olivier, 1789) } \\
\text { Onitini } \\
\text { Bubas bison (Linnaeus, 1767) } \\
\text { Bubas bubaloides Janssens, 1938 } \\
\text { Cheironitis furcifer (Rossi, 1792) } \\
\text { Cheironitis irroratus (Rossi, 1790) } \\
\text { Onitis alexis Klug, 1835 } \\
\text { Onitis belial Fabricius, 1799 } \\
\text { Onitis ion (Olivier, 1789) } \\
\text { Onitis numida Castelnau, 1841 } \\
\text { Onthophagini } \\
\text { Caccobius schreberi (Linnaeus, 1758) } \\
\text { Euonthophagus bedeli (Reitter, 1891) } \\
\text { Euonthophagus crocatus (Mulsant \& Godart, 1870) } \\
\text { Onthophagus atricapillus d'Orbigny, 1908 } \\
\text { Onthophagus fracticornis (Preyssler, 1790) } \\
\text { Onthophagus hirtus (Illiger, 1803) } \\
\text { Onthophagus latigena d'Orbigny, 1897 } \\
\text { Onthophagus maki (Illiger, 1803) } \\
\text { Onthophagus marginalis Gebler, 1817 } \\
\text { Onthophagus melitaeus (Fabricius, 1798) } \\
\text { Onthophagus nebulosus Reiche, 1864 } \\
\text { Onthophagus nigellus (Illiger, 1803) } \\
\text { Onthophagus opacicollis Reitter, 1893 } \\
\text { Onthophagus punctatus (Illiger, 1803) } \\
\text { Onthophagus similis (Scriba, 1790) } \\
\text { Onthophagus sticticus (Harold, 1867) } \\
\text { Onthophagus taurus (Schreber, 1759) } \\
\text { Onthophagus vacca (Linnaeus, 1767) } \\
\end{array}$ \\
\hline & $\begin{array}{l}\text { Psammodiini } \\
\text { Brindalus porcicollis (Illiger, 1803) } \\
\text { Leiopsammodius laevicollis (Klug, 1845) } \\
\text { Psammodius laevipennis (Costa, 1844) }\end{array}$ & & $\begin{array}{l}\text { Scarabaeini } \\
\text { Gymnopleurus flagellatus (Fabricius, 1787) } \\
\text { Gymnopleurus sturmi MacLeay, 1821 } \\
\text { Scarabaeus cicatricosus (Lucas, 1846) } \\
\text { Scarabaeus laticollis Linnaeus, 1767 } \\
\text { Scarabaeus sacer Linnaeus, 1758 } \\
\text { Scarabaeus semipunctatus Fabricius, } 1792 \\
\text { Scarabaeus variolosus Fabricius, 1787 } \\
\text { Sisyphus schaefferi (Linnaeus, 1758) }\end{array}$ \\
\hline
\end{tabular}


Tabla 3.- Subfamilias, tribus y número de especies de Aphodiidae de los distintos géneros conservados en la Colección de Entomología del Museo Nacional de Ciencias Naturales.

Table 3.- Subfamilies, tribes and number of species of different Aphodiidae genera preserved in the Collection of Entomology of the MNCN.

\begin{tabular}{|c|c|c|c|}
\hline Subfamilia & Tribu & Género & $\begin{array}{l}N^{\circ} \text { de } \\
\text { pecies }\end{array}$ \\
\hline \multirow[t]{3}{*}{ Aphodiinae } & Aphod & $\begin{array}{l}\text { Acrossus Mulsant, } 1842 \\
\text { Agrilinus Mulsant \& Rey, } 1870 \\
\text { Ahermodontus Báguena, } 1930 \\
\text { Alocoderus A. Schmidt, } 1913 \\
\text { Amidorus Mulsant \& Rey, } 1870 \\
\text { Ammoecius Mulsant, } 1842 \\
\text { Anomius Mulsant \& Rey, } 1870 \\
\text { Aphodius Illiger, } 1798 \\
\text { Biralus Mulsant \& Rey, } 1870 \\
\text { Bodiloides M. Dellacasa \& G. Dellacasa, } 2005 \\
\text { Bodilopsis Ádám, 1994 } \\
\text { Bodilus Mulsant \& Rey, } 1870 \\
\text { Calamosternus Motschulsky, } 1859 \\
\text { Chilothorax Motschulsky, } 1859 \\
\text { Colobopterus Mulsant, } 1842 \\
\text { Erytus Mulsant \& Rey, } 1870 \\
\text { Eudolus Mulsant \& Rey, } 1870 \\
\text { Euheptaulacus G. Dellacasa, } 1983 \\
\text { Euorodalus G. Dellacasa, } 1983 \\
\text { Eupleurus Mulsant, } 1842 \\
\text { Labarrus Mulsant \& Rey, } 1870 \\
\text { Mecynodes Mulsant \& Rey, } 1870 \\
\text { Megatelus Reitter, } 1892 \\
\text { Melinopterus Mulsant, } 1842 \\
\text { Mendidius Harold, } 1868 \\
\text { Nialosternus Hollande \& Thérond, } 1999 \\
\text { Nialus Mulsant \& Rey, } 1870 \\
\text { Nimbus Mulsant \& Rey, } 1870 \\
\text { Otophorus Mulsant, } 1842 \\
\text { Parabodilus Hollande \& Thérond, } 1999 \\
\text { Phalacronotus Motschulsky, } 1859 \\
\text { Plagiogonus Mulsant, } 1842 \\
\text { Pseudacrossus Reitter, } 1892 \\
\text { Pseuderytus Hollande \& Thérond, } 1999 \\
\text { Pseudesymus d'Orbigny, } 1896 \\
\text { Subrinus Mulsant \& Rey, } 1870 \\
\text { and }\end{array}$ & $\begin{array}{l}1 \\
2 \\
1 \\
2 \\
2 \\
3 \\
4 \\
2 \\
1 \\
1 \\
1 \\
4 \\
4 \\
3 \\
1 \\
1 \\
1 \\
1 \\
3 \\
1 \\
1 \\
2 \\
1 \\
3 \\
1 \\
1 \\
1 \\
2 \\
1 \\
1 \\
1 \\
2 \\
1 \\
1 \\
1 \\
1\end{array}$ \\
\hline & Psam & $\begin{array}{l}\text { diini } \\
\text { Brindalus Landin, } 1960 \\
\text { Leiopsammodius Rakovic, } 1981 \\
\text { Psammodius Fallen, } 1807\end{array}$ & $\begin{array}{l}1 \\
1 \\
1\end{array}$ \\
\hline & Rhyss & $\begin{array}{l}\text { nini } \\
\text { Platytomus Pittino \& Mariani, } 1986 \\
\text { Pleurophorus Mulsant, } 1842 \\
\text { Rhyssemoides Reitter, } 1892 \\
\text { Rhyssemus Mulsant, } 1842\end{array}$ & $\begin{array}{l}2 \\
1 \\
1 \\
3\end{array}$ \\
\hline 1 & 3 & 43 & 70 \\
\hline
\end{tabular}

también contiene varios ejemplares tipo, entre los que destacan: 11 sintipos de Onthophagus taurus var. circumcinctum Escalera, 1914; 1 sintipo de Euorodalus boiteli (Théry, 1918); holotipo y 2 paratipos de Nimbus anyerae Ruiz, 1998; holotipo y 1 paratipo de Thorectes coloni Ruiz, 1998.

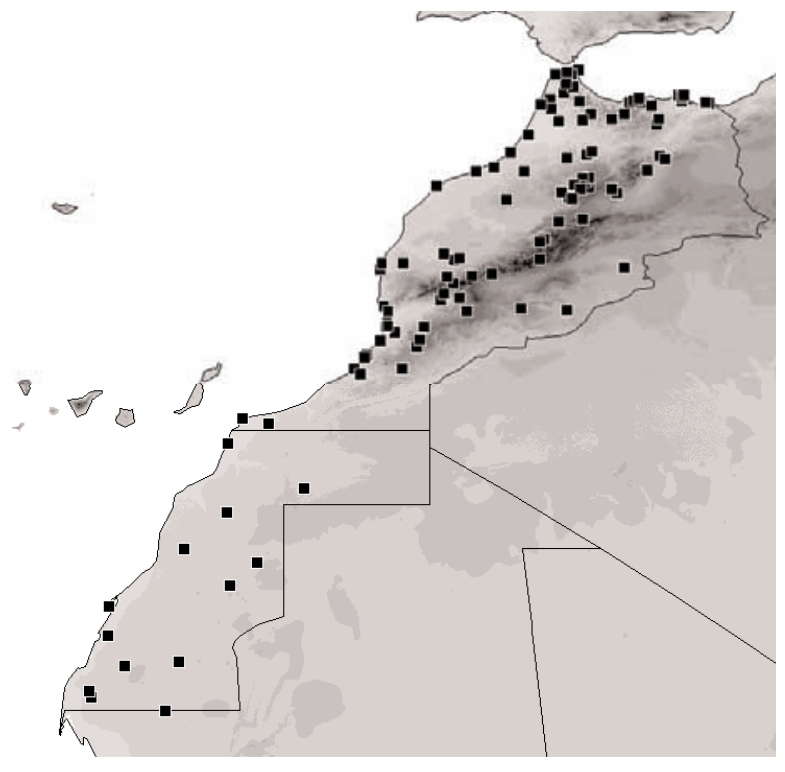

Fig. 2.- Localidades de colecta de Aphodiidae presentes en las colecciones del Museo Nacional de Ciencias Naturales.

Fig. 2.- Localities of collection of Aphodiidae present in the collections of the MNCN.

Las primeras capturas se remontan a 1894 , siendo las últimas del año 2007 (Fig. 1). El primer colector del que se tiene constancia, en la colección estudiada, es M. Le Boul en 1900. Los últimos, en el 2007: Jorge Miguel Lobo, Joaquín Hortal y Alberto Jiménez-Valverde. Desde un punto de vista geográfico, existen ejemplares recolectados en el valle de Atúi, en el antiguo Sáhara español, sobre los $21^{\circ} 36^{\prime}$ de latitud hasta Ceuta $\left(35^{\circ} 53^{\prime}\right)$ y longitudinalmente entre los $1^{\circ} 54^{\prime}$ de Oujda, en la frontera con Argelia y los $14^{\circ} 21^{\prime}$ de Uad Erchane; y, respecto a la altitud, entre el nivel del mar y los 2.050 metros de altitud en la localidad de AinKahla, en el Medio Atlas. Estas georreferenciaciones sugieren una buena representación del grupo en prácticamente todo Marruecos, no así en el Sáhara Occidental, y no siempre para todas las familias, como veremos a continuación.

\section{Resultados}

\section{Familia Aphodiidae}

Este grupo fundamentalmente endocóprido es numéricamente el más representativo en las colec- 
Tabla 4.- Subfamilias, tribus y número de especies de Geotrupidae de los distintos géneros conservados en la Colección de Entomología del Museo Nacional de Ciencias Naturales.

Table 4.- Subfamilies, tribes and number of species of different Geotrupidae genera preserved in the Collection of Entomology of the MNCN.

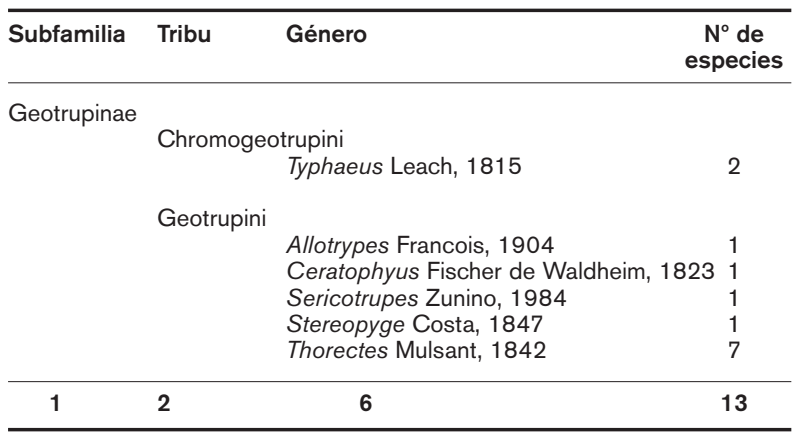

ciones del MNCN. Se conservan 70 especies pertenecientes a 43 géneros (Tabla 3), lo que representa, respectivamente, un $57 \%$ y un $80 \%$ de los afodinos inventariados en Marruecos (122 especies y 54 géneros; ver listado completo en http://www.biogeogra-

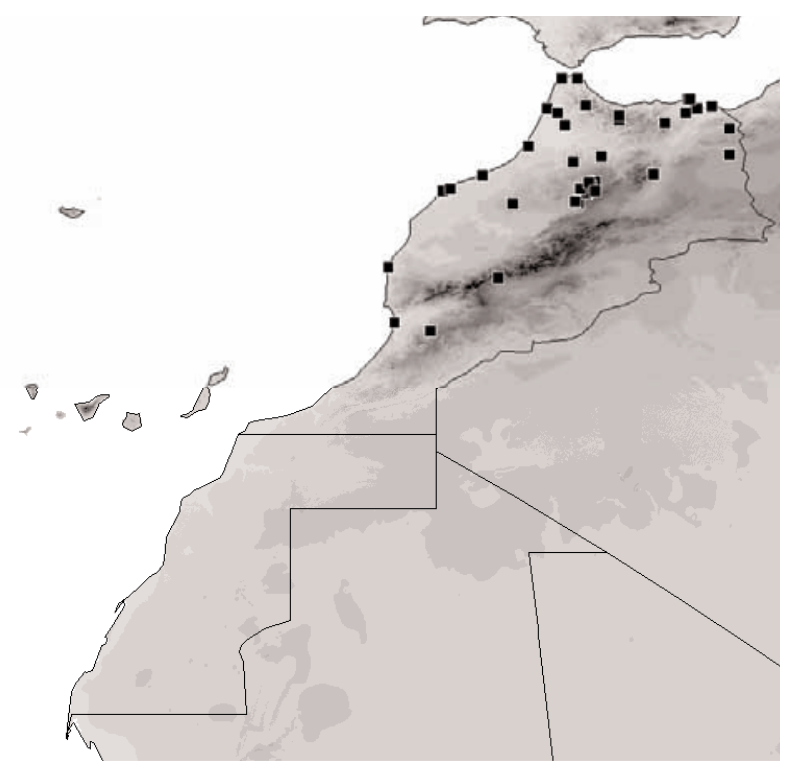

Fig. 3.- Localidades de colecta de Geotrupidae presentes en las colecciones del Museo Nacional de Ciencias Naturales.

Fig. 3.- Localities of collection of Geotrupidae present in the collections of the MNCN.
Tabla 5.- Subfamilias, tribus y número de especies de Scarabaeidae de los distintos géneros conservados en la Colección de Entomología del Museo Nacional de Ciencias Naturales.

Table 5.- Subfamilies, tribes and number of species of different Scarabaeidae genera preserved in the Collection of Entomology of the MNCN.

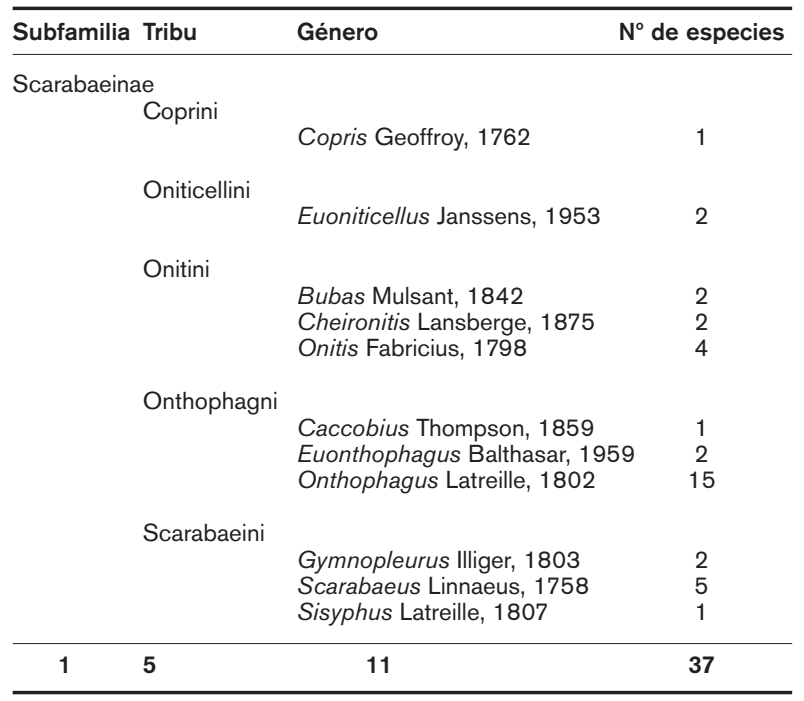

fia.org/). En total se conservan 110.602 ejemplares de esta familia, recolectados por 23 autores en 141 localidades (Fig. 2). En la colección de Entomología del MNCN es la única familia con representantes en el Sáhara Occidental. Cabe reseñar la excelente representación de especies poco comunes en las colecciones, como el caso de Amidorus moraguesi (Baraud, 1978).

\section{Familia Geotrupidae}

El grupo de los geotrúpidos resulta, significativamente, el mejor representado, en cuanto a número de taxones en las colecciones del MNCN. Se conservan 13 especies pertenecientes a 6 géneros (Tabla 4), lo que representa, respectivamente, un $81 \%$ y un $100 \%$ de los geotrúpidos actualmente inventariados en Marruecos (16 especies y 6 géneros; ver listado en http://www.biogeografía.org/). En total, se conservan 664 ejemplares, recolectados por 14 autores en 43 localidades (Fig. 3), ninguna de ellas saharaui.

\section{Familia Scarabaeidae}

El grupo de los escarabeidos está bastante bien representado, tanto en número de ejemplares como 


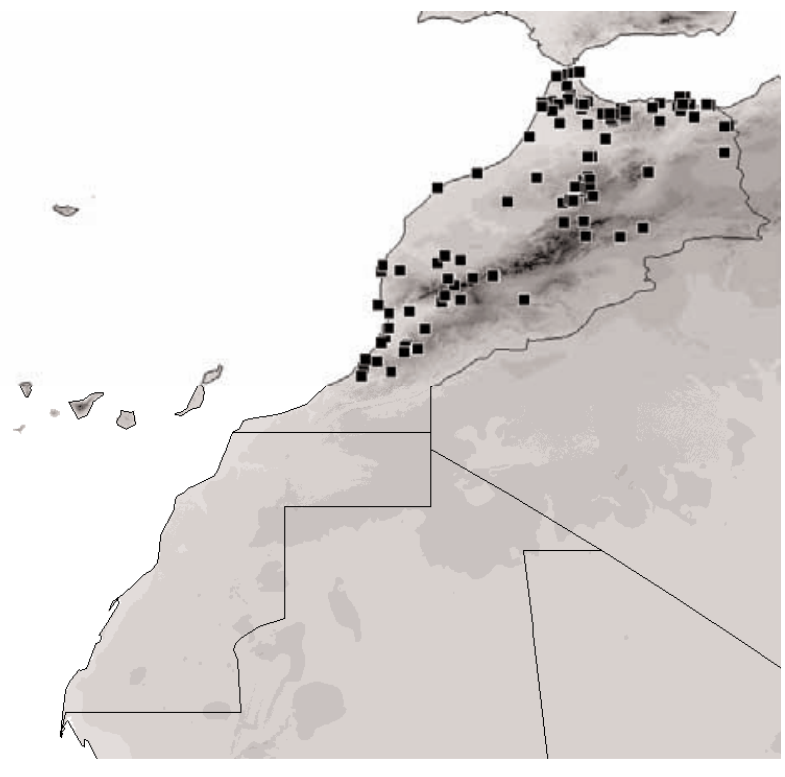

Fig. 4.- Localidades de colecta de Scarabaeidae presentes en las colecciones del Museo Nacional de Ciencias Naturales.

Fig. 4.- Localities of collection of Scarabaeidae present in the collections of the MNCN.

de taxones, en las colecciones del MNCN. Se conservan 37 especies pertenecientes a 11 géneros (Tabla 5), lo que representa, respectivamente, un $79 \%$ y un $92 \%$ de los escarabeidos inventariados en Marruecos (47 especies y 12 géneros). En total se conservan 20.641 ejemplares, recolectados por 32 autores en 85 localidades marroquíes (Fig. 4).

\section{Representatividad y necesidades futuras}

La colección entomológica del MNCN alberga un importante número de ejemplares de Scarabaeoidea Laparosticti coprófagos. La gran mayoría de estos ejemplares (más del 97\%) han sido colectados durante la primera década de este siglo, gracias a las campañas de muestreo dirigidas por el Museo Nacional de Ciencias Naturales, y el resto -básicamente- durante el periodo que va desde principios del siglo pasado hasta la guerra civil española (Fig. 1). Sin embargo, este importante incremento reciente en el número de ejemplares colectados respecto al material histórico disponible, aunque mejo-

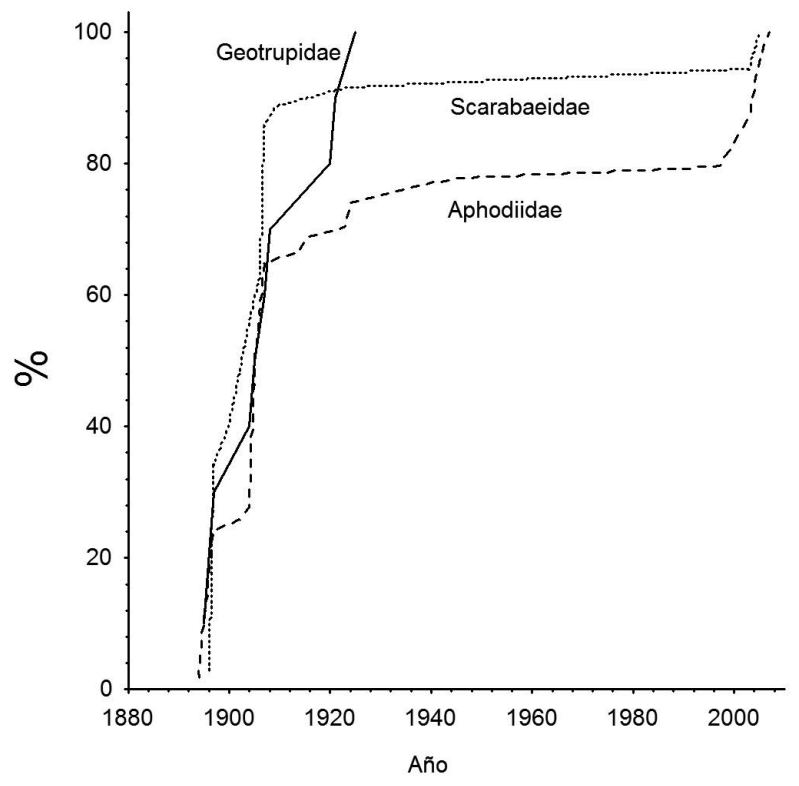

Fig. 5.- Evolución histórica del número de especies que ha albergado la colección de entomología del MNCN para cada una de las familias de Scarabaeoidea coprófagos.

Fig. 5.- Historical evolution of the number of species present in the entomology collection of the MNCN for each family of Scarabaeoidea dung beetles.

ra la representación cuantitativa de estos organismos, no ha significado un aumento notable de la cobertura taxonómica de esta colección (Fig. 5). La totalidad de las especies de Geotrupidae, el 91\% de las especies de Scarabaeidae, y el 74\% de las especies de Aphodiidae disponibles actualmente en el MNCN poseen fechas de colecta anteriores a 1925 y sólo 11 especies de anodinos, 3 de escarabeidos y 1 de geotrupinos se han incorporado a la colección desde el año 2000.

El actual catálogo de Marruecos cuenta con 185 especies (ver listado completo en http://www.biogeografía.org/) y la colección entomológica del MNCN alberga, aproximadamente, un $66 \%$ del total de especies citadas para el territorio marroquí-saharaui $(81 \%$ en el caso de Geotrupidae, $79 \%$ para Scarabaeidae y $57 \%$ en el caso de Aphodiidae). Es evidente, por tanto, que mejorar la representatividad taxonómica de esta colección requerirá, principalmente, realizar intercambios de material o nuevas colectas de las especies de menor talla de la familia Aphodiidae y en las regiones que han sufrido un menor esfuerzo de 


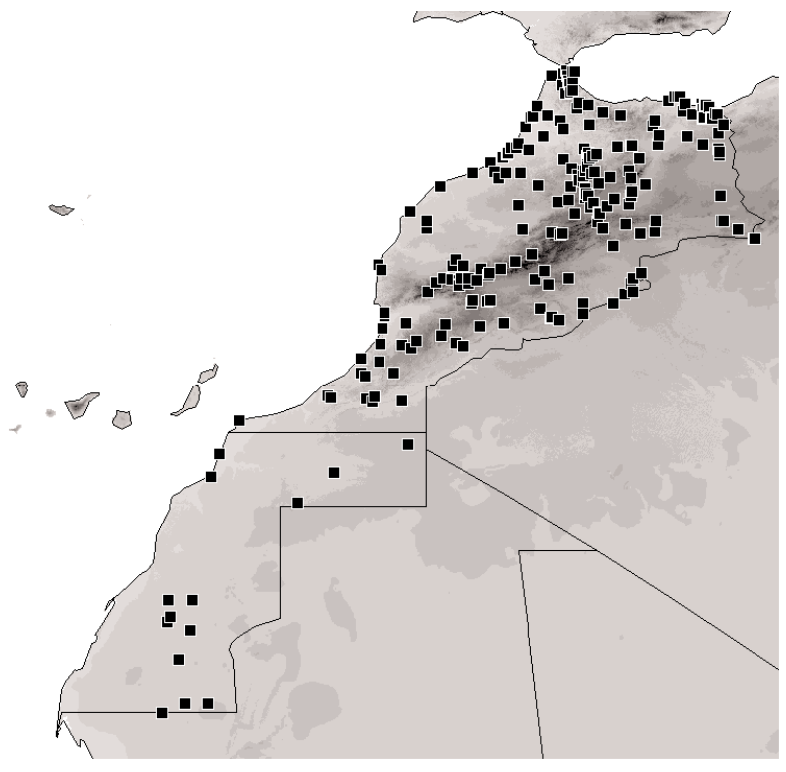

Fig. 6.- Localidades donde han sido citadas especies de Scarabaeoidea coprófagos no presentes en la Colección de Entomología del Museo Nacional de Ciencias Naturales (datos procedentes de la base de datos de Scarabaeoidea coprófagos de Marruecos: http://www.biogeografia.org/).

Fig. 6.- Localities where species of Scarabaeoidea dung beetles not present in the Collection of Entomology of the MNCN have been mentioned (information proceeding from the database of Scarabaeoidea coprophagous of Morocco: http://www.biogeografia.org/).

muestreo (ver áreas sin registros en Figs. 2, 3 y 4) o donde la presencia de otras especies no presentes en dicha colección ha sido constatada (Fig. 6), como en localidades situadas al sur del Medio Atlas, en el Sáhara Occidental o en las áreas limítrofes con Argelia y Mauritania.

\section{Agradecimientos}

A Mercedes París, Amparo Blay y Mercedes Hitado, del Museo Nacional de Ciencias Naturales, por su diligente ayuda en la consulta de las colecciones. A Joaquín Hortal y Alberto Jiménez-Valverde en la realización del trabajo de campo (muestreos de 2003 y 2005). A Fermín Martín Piera (†), quien dos años antes de su fallecimiento ya alentó y participó en el estudio de la fauna de los Scarabaeoidea coprófagos del Magreb. A José Ignacio López-Colón y José Luis Ruiz García por sus acertados comentarios y sugerencias sobre el texto original.

\section{Referencias}

Arias Encobet, J., 1913. Sobre dos Nemestrínidos de Marruecos. Notas dipterológicas. Boletín de la Real Sociedad española de Historia Natural, 13: 150-153, lám. 4.

Baraud, J., 1985. Coléoptères Scarabaeoidea. Faune du Nord de l'Afrique du Maroc au Sinaï. Éditions Lechevalier. París. 651 pp.

Benítez, C., 1899. Mi viaje al interior de África. Imprenta Hispano-arábiga de la Misión-Católica-española. Tánger. 207 pp.

Benítez, C., 2005. Mi viaje al interior de África. Ayuntamiento de Alhaurín de la Torre. Málaga. 207 pp.

Bolívar Pieltain, C. 1930. Una excursión zoológicobotánica por el Rif (Marruecos). Conferencias y Reseñas Científicas, Real Sociedad Española de Historia Natural, 5(3-4): 181-193.

Cabrera, A., 1924. Magreb-el-Aksa. Recuerdo de cuatro viajes por Yebala y por el Rif. Ibersaf Editores. Biblioteca de temas de interés nacional, Volumen 3. Madrid. 270 pp.

Casado, A., 1998. Rumbo a Oriente con Manuel Martínez de la Escalera. Quercus, 151: 41-45.

Cobos, A., 1955. Prospección entomológica en la zona Española del protectorado en Marruecos. Archivos del Instituto de Aclimatación, 4: i-ix.

Cobos, A., 1961. Exploración entomológica del cedral del Tizi-Ifri (Macizo del Iquelmalet Rif central, Marruecos). Archivos del Instituto de Aclimatación, 10: 63-99.

Conde-Salazar Infiesta, L., 2009. Atlas de los exploradores españoles. Sociedad Geográfica Española. Barcelona. 320 pp.

Giner Marí, J., 1944. Resultados científicos de un viaje entomológico al Sáhara español y zona oriental del Marruecos español. Familias Sphecidae y Mutillidae (sensu lato) (Hym. Sphec. y Mut.). Eos, 20: 351385.

González Bueno, A. \& Gomis Blanco, A., 2007. Los territorios olvidados. Naturalistas españoles en el África hispana (1860-1936). Doce Calles, Theathrum Naturae Colección de Historia Natural. Aranjuez. 552 pp.

Hernández-Pacheco, E., Hernández-Pacheco, F., Alia Medina, M., Vidal Box, C. \& Guinea López, E., 1949. El Sáhara español. Instituto de Estudios Africanos, Conseo Superior de Investigaciones Científicas. Madrid. 808 pp.

Hollande, A. \& Thérond, J., 1998. Aphodiidae du Nord de l'Afrique. Monografie XXI. Museo Regionale di Scienzi Naturali di Torino. Torino. 280 pp.

Lobo, J. M., Martín-Piera, F. \& Veiga, C. M., 1988. Las trampas pitfall con cebo, sus posibilidades en el estudio de las comunidades coprófagas de Scarabaeoidea 
(Col.). I. Características determinantes de su capacidad de captura. Revue d'Écologie et Biologie du Sol, 25(1): 77-100.

López-Colón, J. I. \& Baena, M., 2005. Anselmo Pardo Alcaide. Una vida dedicada a la entomología. Consejería de Cultura. Ciudad Autónoma de Melilla. 196 pp.

Martín Alabaladejo, C., 2005. Bibliografía entomológica de autores españoles (1758-2000). Museo Nacional de Ciencias Naturales. Madrid. CD-ROM.

Martínez de la Escalera, M., 1906. Especies nuevas de Coleópteros de Marruecos. Boletín de la Real Sociedad española de Historia Natural, 6: 161-163.

Martínez de la Escalera, M., 1913. Descripción de los coleópteros recogidos en ella (en la campaña entomológica en el Sus). Trabajos del Museo Nacional de Ciencias Naturales, Serie Zoología, 8: 29-56.

Martínez de la Escalera, M., 1914. Los coleópteros de Marruecos. Trabajos del Museo Nacional de Ciencias Naturales, Serie Zoología, 11: 1-553.

Martínez de la Escalera, M., 1925. Especies nuevas de Lamelicornios de Marruecos. Boletín de la Real Sociedad española de Historia Natural, 25: 422-436.

Martínez de la Escalera, M., 1926. Las Hymenoplia de España y Marruecos (Col. Lam.). I. De la validez específica de H. Miegii Gr. y de sus aliadas. Boletín de la Real Sociedad española de Historia Natural, 26: 355-359.

Martínez de la Escalera, M., 1931. Una nueva especie de Elaphocera Gené de Marruecos (Col. Scarab.). Boletín de la Real Sociedad española de Historia Natural, 31: 715-717.

Martínez de la Escalera, M., 1933. Las Hymenoplia Eschz. de Marruecos (Col. Scarab.). Eos, 9: 5-16.

Martínez de la Escalera, M., 1934. Una Elaphocerida Rttr. nueva del Rif (Col. Scarab.). Boletín de la Real Sociedad española de Historia Natural, 34: 129-131.

Mateu, J., 1950. Escarabeidos de Ifni y Sáhara español. Eos, 2: 271-297.

Morales Agacino, E., 2001. Memorias de un naturalista. Organismo Autónomo de Parques Nacionales, Ministerio de Medio Ambiente. Madrid. 274 pp.

Pardo Alcaide, A., 1950. Contribución al conocimiento de la fauna entomológica marroquí III. Publicaciones fuera de serie, Instituto General Franco de Estudios e Investigación Hispano-Árabe. Editorial Marroquí. Tetuán. 74 pp.

Pardo Alcaide, A., 1955. Contribución al conocimiento de la fauna entomológica marroquí, VI. Coleópteros del Valle inferior del Uad Muluya. Tamuda, 3: 39-74.

Pardo Alcaide, A., 1958. El género Glaresis en Marruecos (Col., Trogidae) (VII) Contribución al conocimiento de la fauna entomológica marroquí). Eos, 34: 161-168.
Quiroga, F., 1886. Apuntes de un viaje por el Sáhara Occidental. Anales de la Sociedad española de Historia Natural, 15 : 495-523.

Real Sociedad Española de Historia Natural, 1914. Yebala y el Bajo Lucus. Sociedad Española de Historia Natural. Madrid. 315 pp.

Romero-Samper, J., 2008. Las comunidades de coleópteros Escarabeidos coprófagos (Coleoptera, Scarabaeoidea) del Medio Atlas (Marruecos): influencia del tipo de hábitat, altitud y estacionalidad. Análisis comparado de su estructura. Tesis Doctoral. Universidad Complutense de Madrid. Madrid. 357 pp. + VII anexos.

Veiga, C. M., Lobo, J. M. \& Martín-Piera, F., 1989. Las trampas pitfall con cebo, sus posibilidades en el estudio de las comunidades coprófagas de Scarabaeoidea (Col.). II. Análisis de efectividad. Revue d'Écologie et Biologie du Sol, 26(1): 91-109.
Recibido / Received, 3-03-2011 Aceptado / Accepted, 9-05-2011 Publicado en línea / Published online, 1-06-2011 Publicado impreso / Published in print, 30-06-2011 